\section{Endoscopic ultrasound-guided pancreatic duct drainage using antegrade stenting}

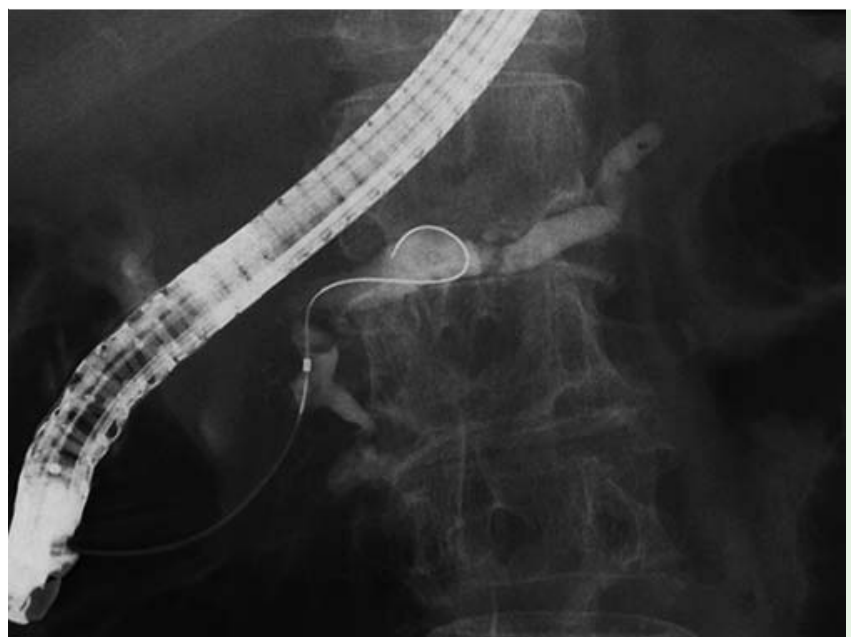

Fig. 1 Endoscopic ultrasound (EUS)-guided pancreatic duct antegrade stenting was performed on a 66-year-old woman. At endoscopic retrograde cholangiopancreatography (ERCP) the guidewire was successfully advanced into the pancreatic duct through the stenosis, but the ERCP cannula and other devices, including the dilation catheter, could not be advanced.

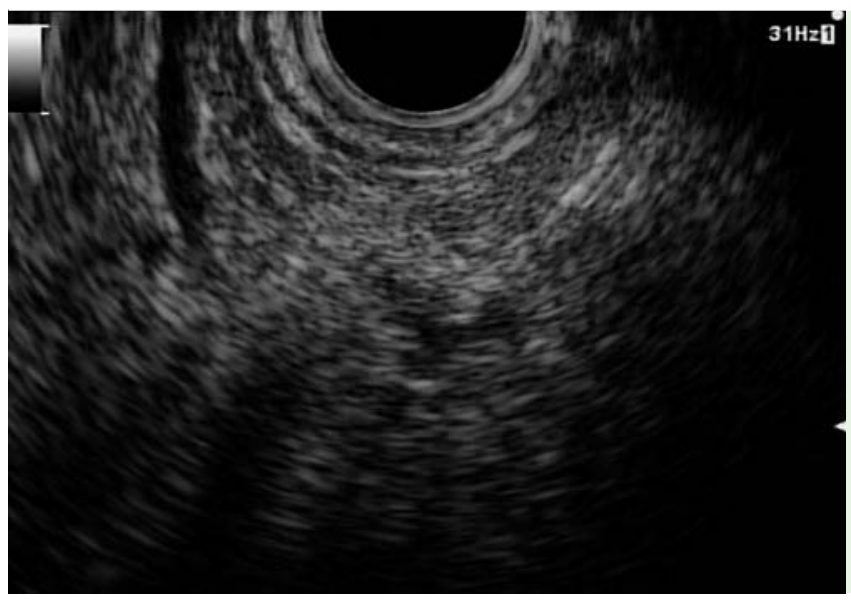

Fig. 2 The pancreatic duct has been punctured using a 19-G needle (endosonographic image).

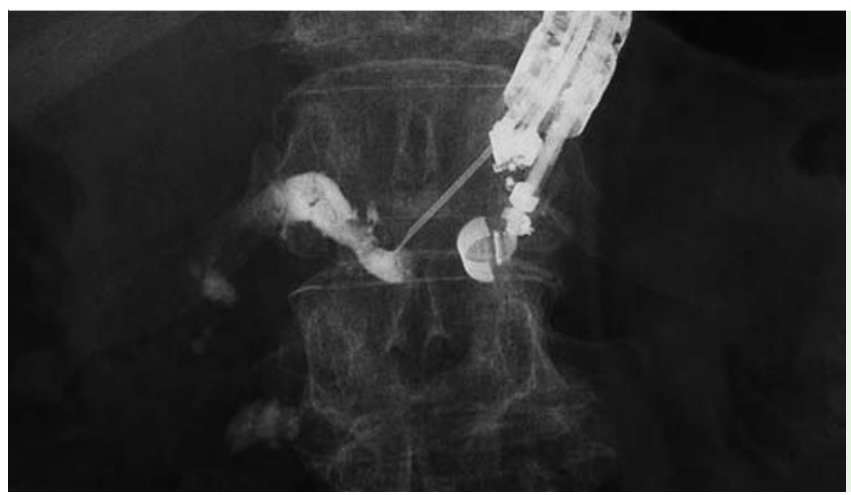

Fig. 3 The contrast medium was injected through the 19-G needle.

Endoscopic ultrasound (EUS)-guided pancreatic duct access in patients with an inaccessible papilla or failed endoscopic retrograde cholangiopancreatography (ERCP) has been reported previously [1, 2]. This technique consists of the EUSguided rendezvous technique, and EUSguided pancreatic duct stenting via the duodenostomy, and it may easily occur because of food impaction. Novel technical tips for EUS-guided pancreatic duct antegrade stenting are presented here (๑ Video 1 ).

A 66-year-old woman was admitted to our hospital because of abdominal pain. She had previously had chronic pancreatitis. On magnetic resonance cholangiopancreatography (MRCP) and EUS imaging, the pancreatic duct was observed to be dilated from the head of the pancreas, around which a pancreatic stone was also seen. This pancreatic stenosis was treated using a pancreatic stent. First, we performed ERCP, through which we observed the stenosis of the pancreatic duct. However, we were unable to pass the ERCP cannula through the stenosis site ( $\bullet$ Fig. 1 ). Next, the scope was changed from a duodenoscope to a convex echoendoscope. To avoid any intervening vessels, the pancreatic duct was first punctured using a 19-G needle under Doppler imaging ( $\bullet$ Fig. 2). After pancreatic juice had been aspirated, contrast medium was injected, and images of the pancreatic duct were obtained ( Fig.3). Then, a 0.025-inch stiff guidewire was inserted, and was easily advanced into the duodenum through the stenosis. Next, the 19-G needle was exchanged for an ERCP catheter to dilate the fistula ( $\bullet$ Fig.4). Although an EUS-guided rendezvous approach was considered, EUS-guided antegrade stenting of the pancreatic duct was attempted because no leakage of pancreatic juice was seen on endosonographic imaging. The 7-Fr straight plastic stent was inserted antegradely, from the duodenum to the pancreatic duct ( $\bullet$ Fig.5). No adverse events were seen.

Although validation is required in a prospective clinical trial, this novel technique appears to be safe and effective as an option for EUS-guided pancreatic duct drainage.

\section{Video 1}

During endoscopic ultrasound (EUS)-guided pancreatic duct antegrade stenting, the main pancreatic duct is punctured using a 19-G needle, and contrast medium is injected. A 0.025 -inch guidewire is inserted through the 19-G needle and advanced into the duodenum. To dilate the fistula, a cannula used for endoscopic retrograde cholangiopancreatography (ERCP) is inserted. Finally, a 7-Fr plastic stent is placed antegradely from the duodenum to the pancreatic duct. 

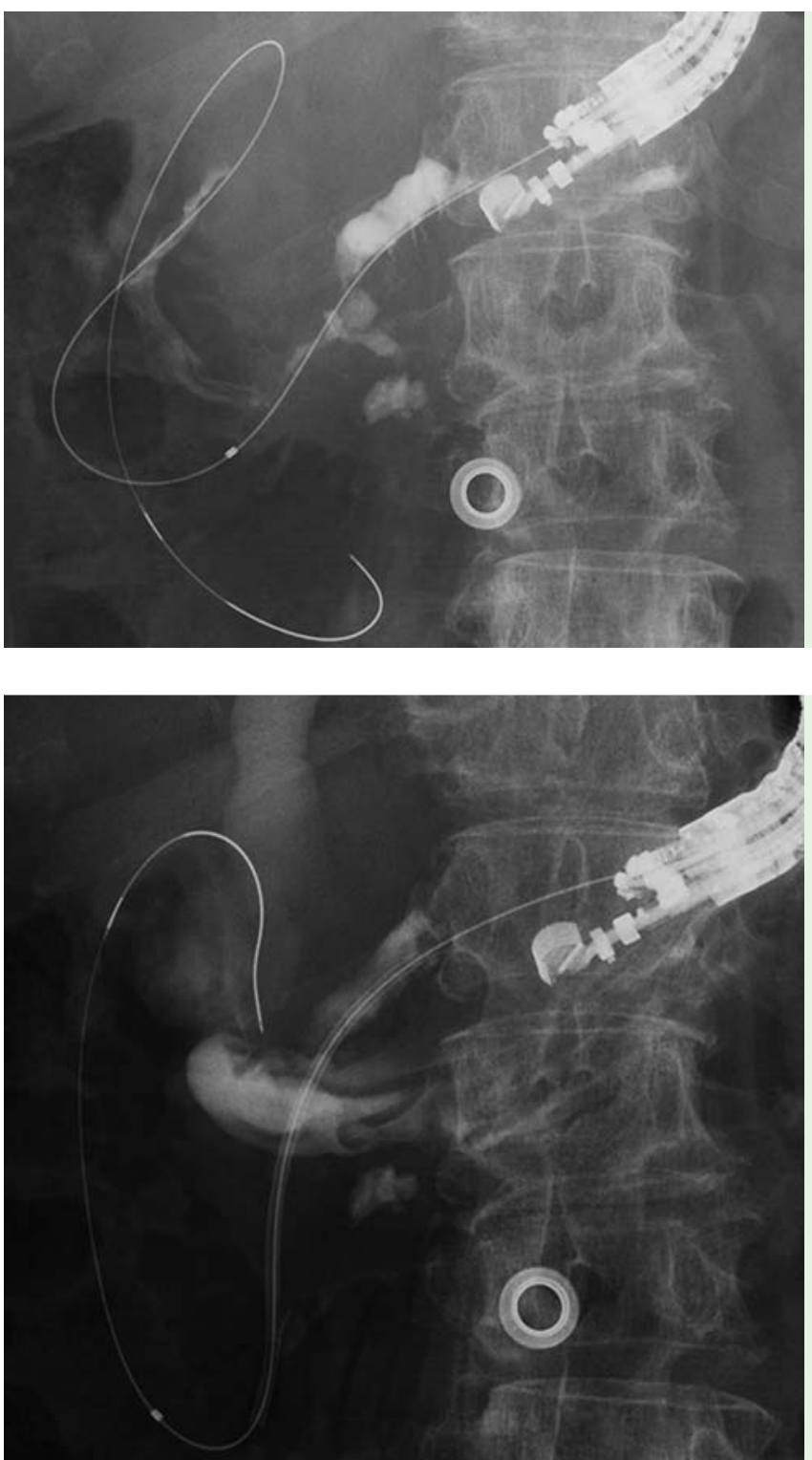

Fig. 4 A 0.025-inch guidewire and ERCP cannula have been easily advanced into the duodenum through the stenosis.

Endoscopy_UCTN_Code_TTT_1AS_2AD

Competing interests: None

Takeshi Ogura', Saori Onda', Tatsushi Sano ${ }^{1}$, Kazuhiro Yamamoto ${ }^{2}$, Daisuke Masuda ${ }^{1}$, Akira Imoto', Kazuhide Higuchi ${ }^{1}$

${ }^{1}$ Second Department of Internal Medicine, Osaka Medical College, Osaka, Japan

2 Department of Radiology, Osaka Medical College, Osaka, Japan

\section{References}

1 Kahaleh M, Hernandez AJ, Tokar J et al. EUSguided pancreaticogastrostomy: analysis of its efficacy to drain inaccessible pancreatic ducts. Gastrointest Endosc 2007; 65: $224-$ 230

Fig. 5 A 7-Fr plastic stent has been placed antegradely.

2 Itoi T, Yasuda I, Kurihara T et al. Technique of endoscopic ultrasonography-guided pancreatic duct intervention (with video). J Hepatobiliary Pancreat Sci 2014; 21: E4 -E9

3 Giovannini M. Endoscopic ultrasonographyguided pancreatic drainage. Gastrointest Endosc Clin N Am 2012; 22: 221 -230

\section{Bibliography}

DoI http://dx.doi.org/

10.1055/s-0034-1391422

Endoscopy 2015; 47: E161-E162

(c) Georg Thieme Verlag KG

Stuttgart · New York

ISSN 0013-726X

\section{Corresponding author}

\section{Takeshi Ogura, PhD, MD}

Second Department of Internal Medicine

Osaka Medical College

1-1 Daigakuchou, Takatsukishi

Osaka 464-8681

Japan

Fax: +81-52-7635233

oguratakeshi0411@yahoo.co.jp 